\title{
Computational Search for Strong Topological Insulators: An Exercise in Data Mining and Electronic Structure
}

\author{
M. Klintenberg ${ }^{1}$, J. T. Haraldsen ${ }^{2,3,4}$ \& A. V. Balatsky ${ }^{5,6}$ \\ ${ }^{1}$ Department of Physics and Astronomy, Uppsala University, Box 516, SE, Uppsala, Sweden \\ 2 Department of Physics and Astronomy, James Madison University, Harrisonburg, VA, USA \\ 3 Theoretical Division, Los Alamos National Laboratory, Los Alamos, NM, USA \\ ${ }^{4}$ Center for Integrated Nanotechnologies, Los Alamos National Laboratory, Los Alamos, NM, USA \\ ${ }^{5}$ Institute for Material Science, Los Alamos National Laboratory, Los Alamos, NM, USA \\ ${ }^{6}$ NORDITA, Roslagstullsbacken 23, 10691 Stockholm, Sweden
}

Correspondence: J. T. Haraldsen, Department of Physics and Astronomy, James Madison University, Harrisonburg, VA 22807, USA. E-mail: haraldjt@jmu.edu

\author{
Received: April 16, 2014 Accepted: May 8, 2014 Online Published: June 19, 2014 \\ doi:10.5539/apr.v6n4p31 URL: http://dx.doi.org/10.5539/apr.v6n4p31
}

\begin{abstract}
We report a data-mining investigation for the search of topological insulators by examining individual electronic structures for over 60,000 materials. Using a data-mining algorithm, we survey changes in band inversion with and without spin-orbit coupling by screening the calculated electronic band structure for a small gap and a change concavity at high-symmetry points. Overall, we were able to identify a number of topological candidates with varying structures and composition. Our overall goal is expand the realm of predictive theory into the determination of new and exotic complex materials through the data mining of electronic structure.
\end{abstract}

Keywords: electronic structure, data mining, topological insulators, predictive theory

\section{Introduction}

Over the past few years, materials theory has moved towards better predictive capabilities help with materials understanding and possible design. This has lead to numerous projects including the materials gnome initiative (Materials Genome Initiative for Global Competitiveness, 2014; Yang, Setyawan, Wang, Buongiorno Nardelli, \& Curtarolo, 2012) focusing on predictive theory and an effective understanding of complex materials (Klintenberg, Derenso, \& Weber, 2002a, 2002b; Klintenberg, 2010, 2014; Klintenberg \& Eriksson, 2013; Lebegue, Bj orkman, Klintenberg, Nieminen, \& Eriksson, 2013; Ortiz, Eriksson, \& Klintenberg, 2009). This would include everything from phenomenological approaches to various techniques including density functional theory (DFT) and dynamical mean field theory (DMFT) have been used to further this understanding (Klintenberg \& Eriksson, 2013; Lebegue, Bj orkman, Klintenberg, Nieminen, \& Eriksson, 2013; Ortiz, Eriksson, \& Klintenberg, 2009; Klintenberg, 2014; Fisher, Tibbetts, Morgan, \& Ceder, 2006; Seshadri, Brock, Ramirez, Subramanian, \& Thompson, 2012; Morales, McMahon, Pierleoni, \& Ceperley, 2013; Benyelloul \& Aourag, 2013; Olson, 2013). The use of predictive algorithms provides the potential to accelerate the discovery and identification of new materials. The advancement of these algorithms for calculating the electronic structure can be coupled to data-mining techniques and used to screen or search for exotic materials or phases like the topological insulators.

Topological insulators (Moore, 2010; Hasan \& Kane, 2010; Kane£ 2008; Buttiker, 2009; Moore, 2009a, 2009b) are a new quantum phase of matter with exotic properties (such as nearly dissipationless transport and protection against Anderson localization) (Roushan et al., 2009). These new states of quantum matter could enable the realization of quantum computing (Collins, 2003; Bonderson, Sarma, Freedman, \& Nayak, 2010) and will probably result in new spintronic and magnetoelectric devices. Because of these potential technological applications, topological insulator research has gained a considerable amount of momentum over the last few years.

The prediction and realization of the 3D topological insulators (Lin et al., 2010; Fu, Kane, \& Mele, 2007; Moore 
\& Bals, 2007; Roy, 2009; Hsieh et al., 2008; Hsieh, 2009; Xia, 2009; Zhang, 2009; Chen, 2009) grew out of the work on the quantum Hall effect and quantum spin Hall effect in 2D (Murakami, Nagaosa, \& Zhang, 2004; Kane \& Mele, 2005a, 2005b) and the essence of these central articles (Fu, Kane, \& Mele, 2007; Moore \& Bals, 2007; Roy, 2009; Hsieh et al., 2008; Hsieh, 2009; Xia, 2009; Zhang, 2009; Chen, 2009; Murakami, Nagaosa, \& Zhang, 2004; Kane \& Mele, 2005a, 2005b) is captured in a number of letters describing topological insulators (Moore, 2009a, 2009b, 2010; Kane, 2008; Buttiker, 2009).

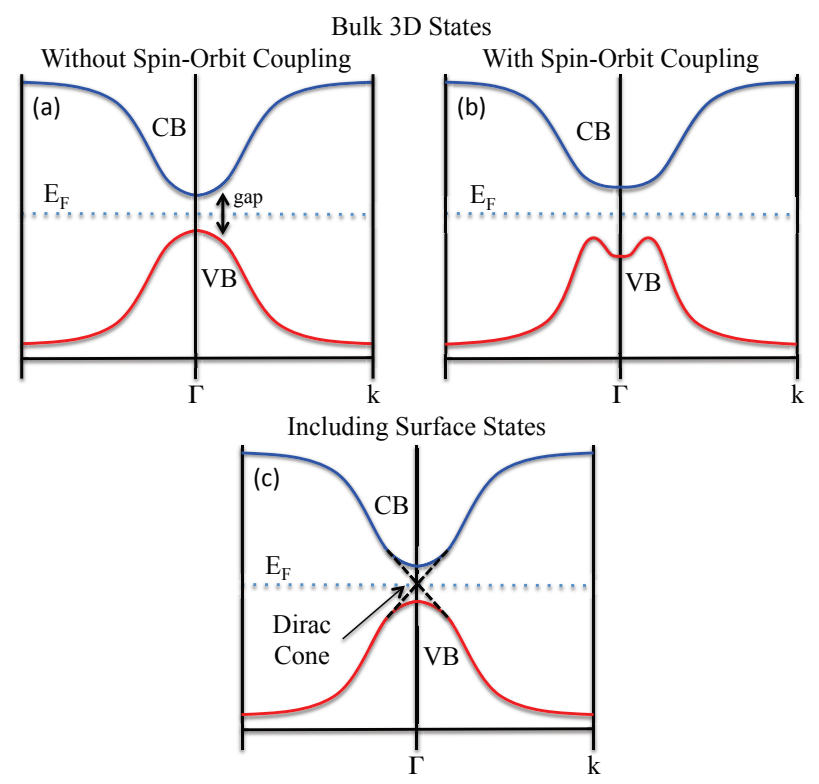

Figure 1. Band inversion because of strong spin-orbit coupling in small electronic band gap materials can be identified by searching for the anti-crossing feature around the $\Gamma$-point without spin-orbit coupling (a) or with spin-orbit interaction (b). If one were to include surface states, then the presence of a Dirac cone would appear (c)

Strong topological insulators in three dimensions can be viewed as insulators in the bulk, but with metallic surface states. These surface states resemble the Dirac cones observed graphene (illustrated in Figure 1), and they exhibit remarkable properties such as dissipationless transport, topological protection against perturbations, as well as impurity scattering. Therefore, it is not surprising that these new materials have spawned such great attention and research in the science community.

An insulator is typically characterized by its inability to conduct electrons (i.e. an external electric field does not cause current flow). Alternatively, an insulator is a system that lacks low-lying excited states and takes a finite amount of energy for an electronic excitation (i.e. we have an electronic band gap). From this, it appears that the electronic band gap is the one characteristic needed to determine an insulator. However, the discovery of the quantum Hall $(\mathrm{QH})$ effect changed this claim. The $\mathrm{QH}$ state appears when a two-dimensional electron gas is subject to a magnetic field. The interior of the $\mathrm{QH}$ state has a band gap, but contrary to a normal insulator, the $\mathrm{QH}$ state has edge states that do not show a band gap, which means there is a current on the boundary of the sample. This charge flow is one directional, which protects it against backscattering (Hasan \& Kane, 2010). Both the insulating and QH state systems show bulk band gaps, but the latter also has the surface states that are insensitive to scattering. Therefore, the difference between the two systems is the topology of the occupied bands, or more specifically, the topological class of the bulk band structure.

In 2005, the quantum spin Hall (QSH) effect was discovered (Kane \& Mele, 2005a, 2005b), where the QSH state is characterized by another topological invariant (knot) and can exist without a magnetic field (a QH state required a magnetic field) due to the spin-orbit interaction. In these materials, the bulk has a band gap, while the edge states remain gapless. In graphene, these edge states are spin-filtered and make the different spins travel in opposite directions. The QSH effect can give us edge states without a magnetic field in $2 \mathrm{D}$ because of the spin-orbit interaction. However, the effect in 3D is more complex and provide a more exotic phenomena.

Recently, it has been both predicted and experimentally verified that topological insulators do exist in 3D and that the spin-orbit interaction is the driving force (Fu, Kane, \& Mele, 2007; Moore \& Bals, 2007; Roy, 2009; Hsieh 
et al., 2008, 2009; Xia, 2009; Zhang, 2009; Chen, 2009). We can think of these edge states in 3D topological insulators as a higher dimensional version of the 1D edge states in the QSH state. In 3D, the simplest form of edge state can be viewed as a Dirac fermion metal with a linear excitation energy, c.f. the energy spectrum of massless Dirac fermions. Furthermore, since these surface states are topologically protected by time-reversal symmetry, Anderson localization does not occur even under strong disorder. A topological insulator is simply not allowed to lose the metallic surface state, which means it cannot become gaped or localize. This was experimentally verified for $\mathrm{Bi}_{x} \mathrm{Sb}_{1-x}$ (Roushan, 2009). Although, a gapped state may occur when there exists symmetry breaking or the presence of topological orders.

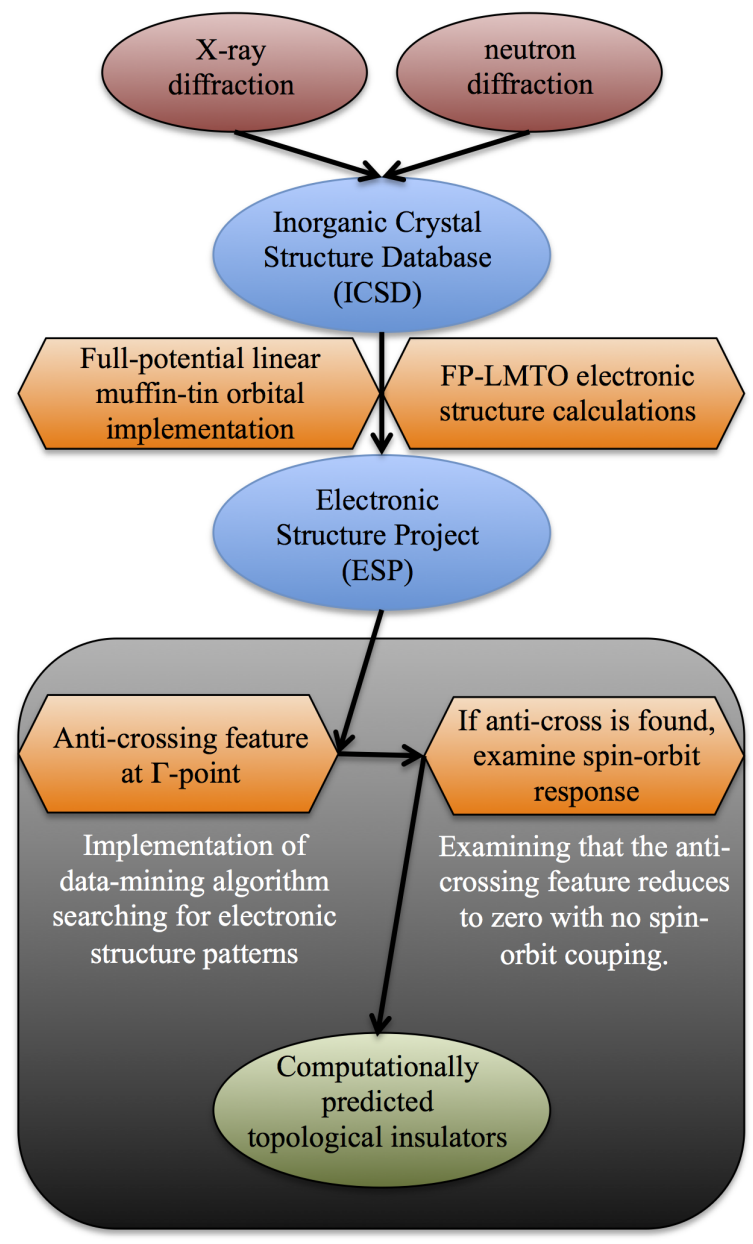

Figure 2. A schematic description of the search for new topological insulators. The key ingredient is the electronic structure project (ESP) that contain the electronic structures of tens of thousands of inorganic compounds. Data mining algorithms on the ESP has the potential to identify a variety of new functional materials

Motivated by the fact that to date only few 3D topological systems have been identified (Lin, 2010; Fu, Kane, \& Mele, 2007; Moore \& Bals, 2007; Roy, 2009; Hsieh et al., 2008, 2009; Xia, 2009; Zhang et al., 2009b; Chen, 2009), we have used massive computing in combination with data-mining algorithm to search for new strong topological insulators (detailed in Figure 2). In this report, we present a number of non-layered (bulk) compounds that show band inversion at the $\Gamma$-point or other $k$-points, a clear signal of a strong topological insulator. We use a data-mining technique to determine the possibility of a topological insulator by screening the electronic structures for different materials and searching for changes in band inversion with and without spin-orbit coupling. Using an algorithm to look for a small gap and a change concavity (a "dip") in the band structure at high-symmetry points (mainly the $\Gamma$-point), we were able to identify a modest, but significant number of topological candidates. Through this computational method, we determine 17 candidates with a variety of structures and composition as strong topological insulators. While our general method only identified a small number of topological insulators, due to the narrow window of parameters used, we find this method could be adapted for a larger data-mining effort. 
However, this effort the strength of using general parameters to further the knowledge of materials through data mining. Since the initial release of these results (Klintenberg, 2010), a number of candidates have been investigated further and some have been confirmed experimentally, which provides validity to the approach of data mining in the search for new and exotic materials.

\section{Methods}

\subsection{Data-Mining of Topological Insulators}

The more then 60,000 electronic structures used in the present study were calculated using a highly accurate fullpotential linear muffin-tin orbital (FP-LMTO) (Skriver, 1984) implementation of density functional theory (DFT) within the local density approximation (LDA). In essence, DFT reduces the many-body problem of solving the electronic structure of some $10^{23}$ interacting electrons into to a one-body problem, thus making the calculation possible. A more detailed description can be found in (Ortiz, Eriksson, \& Klintenberg, 2009; Skriver, 1984).

When data-mining electronic structures in the search for new strong topological insulators, the most straight forward method is to identify materials with a negative band gap (Zhang et al., 2009b). To put it differently, the electronic structure should develop an anti-crossing feature at the $\Gamma$-point when the spin-orbit interaction is turned on as compared to no spin-orbit coupling. A typical band-inversion is shown in Figure 1. Thus, the data-mining algorithm is instructed to search for small gaped materials with an anti-crossing feature at the $\Gamma$-point or other $k$-points. It should be noted that the data-mining of electronic structures have been calculated with spin-orbit coupling.

To show proof-of-concept, the first three known second generation topological insulators $\mathrm{Bi}_{2} \mathrm{Te}_{3}, \mathrm{Bi}_{2} \mathrm{Se}_{3}$ and $\mathrm{Sb}_{2} \mathrm{Te}_{3}$ (Xia, 2009; Zhang et al., 2009b; Chen, 2009) were investigated for band-inversion when spin-orbit interaction was turned on and then off. Indeed, all three materials develop the characteristic anti-crossing feature as spin-orbit coupling is included in the electronic structure calculation. Similar efforts have also been used to look for specific descriptors in electronic structure (Yang, Setyawan, Wang, Buongiorno Nardelli, \& Curtarolo, 2012). The advantage to using these methods is to provide relatively fast identification of candidate compounds. In further studies, one could use other variations of DFT calculations (Generalized Gradient Approximations (GGA), on-site Hubbard potentials, etc.) to probe deeper into the fine points of the electronic structure.

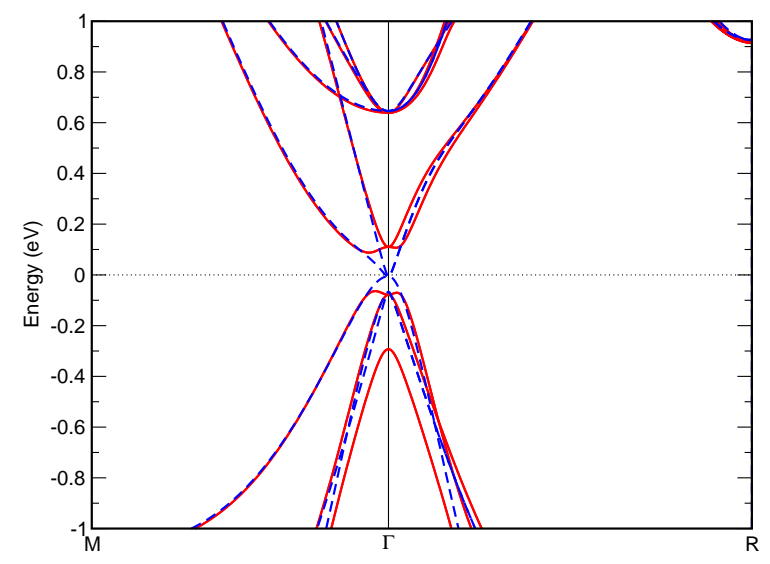

Figure 3. $\mathrm{Ca}_{3} \mathrm{SnO}$ is an example of inverse perovskite that show band inversion. The material transforms from direct gap (no spin-orbit interaction included, blue-dashed line) at the $\Gamma$-point to in-direct gap (around the same point in inverse space) when the spin-orbit is included (red-solid line) in the electronic structure calculation, i.e. the upper most valence band is pushed way and the anti-crossing feature appears. The LDA band gap is $0.2 \mathrm{eV}$ which qualifies the compound for operation under ambient conditions

Our general data-mining algorithm is shown in Figure 2. In general, we start with bulk material crystal structures provided by the inorganic crystal structure database (ICSD), which is a collection of 160,000 experimentallydetermined crystal structures obtained by X-ray and neutron diffraction. Using only the crystal structure as input, 
we have calculated the electronic structure for about 60,000 entries in the ICSD using a full-potential linear muffintin orbital implementation (FP-LMTO) (Skriver, 1984) of density functional theory (DFT) within the local density approximation (LDA). The electronic structures are then deposited and maintained within the electronic structure project (ESP) and has been made available on the web (Ortiz, Eriksson, \& Klintenberg, 2009; Klintenberg, Derenso, \& Weber, 2002a, 2002b). From this point, the data-mining process in the search for new strong topological insulators is rather straight forward. Non-layered (bulk) small gap materials $(\leq 0.5 \mathrm{eV})$ with an anti-crossing feature at the $\Gamma$-point are identified by looking for crossover features at the Fermi level. Furthermore, the topological state is then computationally verified by examining that the anti-crossing feature reduced to zero in the limit the spin-orbit interaction is zero. If this occurs, then we label the material as a potential candidate for having topological states. Experimental observations are then needed for complete determination. While we only identified 17 candidates out of 60,000 initial structure, a more detailed and finely tuned data-mining effort will be able to find more materials that fit these criteria.

\section{Results}

Present computational capabilities do open the new route of computer-assisted materials search that opens up new avenues for materials discovery and informed search. The approach we use is centered on a search of specific band structure feature that would be an indicator of a band inversion. We do point out that this approach is general and does allow one to use it in other contexts. Recent interest in "materials genome" approach (Materials Genome Initiative for Global Competitiveness; Klintenberg M. Electronic Structure Database) is another example of the new ear of a search of new materials using computerized approaches and sophisticated search algorithms.

While this method is general, the data-mining of calculated structure patterns identifies 17 compounds as potential strong topological insulators (presented in Table 1). Originally, these results were submitted to the condensed matter ArXiv for experimental verification (Klintenberg, 2010). Since that time, a number of these materials have been investigated further and some have been verified by experiment or further theoretical investigation. These references are given in the last column of Table 1. Note that because the ESP takes crystal structures from the ICSD (Klintenberg M. Electronic Structure Database), all compounds identified in this study exist and have been structurally determined.

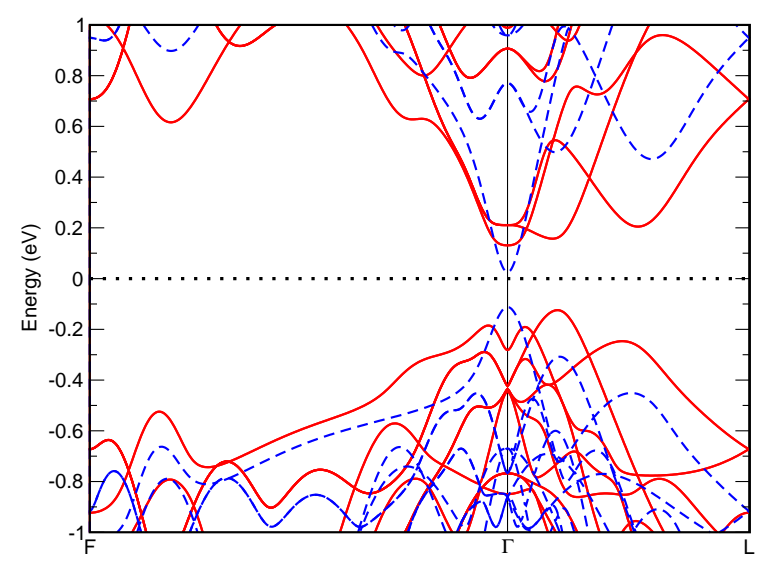

Figure 4. $\mathrm{Bi}_{2} \mathrm{STe}_{2}$ has the same structure type as the celebrated topological insulator $\mathrm{Bi}_{2} \mathrm{Te}_{3}$. We see the same effect of the spin-orbit interaction as in the $\mathrm{Ca}_{3} \mathrm{OSn}$ case. The valence band at the $\Gamma$-point is pushed away and the anti-crossing feature show up when the spin-orbit interaction is included (red-solid line, blue-dashed line for no spin-orbit interaction). This compound could work under ambient conditions because of the $0.3 \mathrm{eV}$ band gap

The first seven materials in Table 1 belong to the $\mathrm{CaTiO}_{3}$ (perovskite) structure type, but are inverse-perovskites. Perovskites are interesting materials because they host a variety of materials properties such as superconductivity, spin dependent transport, ferroelectricity, and colossal magnetoresistance (Hazen, 1988). Present calculations indicate that the strong topological insulator phase now can be added to this list. Through out the Brillouin zone 
these materials have an in-direct LDA band gap ranging from 0.1 to $0.2 \mathrm{eV}$. The characteristic anti-crossing feature is present at the $\Gamma$-point and if the spin orbit coupling constant is set to zero the anti-crossing feature disappears. From the partial density of sates, we conclude that these bands have mainly $p$-character. The electronic band structure for $\mathrm{Ca}_{3} \mathrm{OSn}$ is shown in Figure 3.

The next material in Table 1 is the half-Heusler compound GdPtSb with a AlLiSi structure type. The half Heusler family has been discussed in Ref. (Lin et al., 2010), but it exhibits a distorted structure. Here, it is explicitly demonstrated for GdPtSb that with the experimental structure the material develops an anti-crossing feature when the spin-orbit term is included in the calculations. We speculate that this is also true for the other materials in (Lin et al., 2010). GdPtSb has an indirect band gap of $0.2 \mathrm{eV}$.

$\mathrm{Bi}_{2} \mathrm{SeTe}_{2}$ and $\mathrm{Bi}_{2} \mathrm{STe}_{2}$ both have the $\mathrm{Bi}_{2} \mathrm{Te}_{3}$ structure type. In fact, these materials can be viewed as $\mathrm{Bi}_{2} \mathrm{Te}_{3}$ but with every third Te replaced with $\mathrm{Se}$ and $\mathrm{S}$, respectively. $\mathrm{Bi}_{2} \mathrm{Te}_{3}$ is a celebrated second generation topological insulator (Zhang et al., 2009b) and both the Se and S variations have similar electronic structure to $\mathrm{Bi}_{2} \mathrm{Te}_{3}$ and show the anti-crossing feature at the $\Gamma$-point when spin-orbit interaction is removed. The indirect band gaps are $0.3 \mathrm{eV}$, respectively. The electronic band structure for $\mathrm{Bi}_{2} \mathrm{STe}_{2}$ is shown in Figure 4.

Table 1. Results of the mining algorithm for second generation non-trivial topological insulators. 17 compounds are identified as new potential topological insulators. ARPES measurements should confirm our findings. The materials that have been either confirmed or investigated further are cited in the last column. The electronic band structures, partial and total density of states are given for all materials in the supplementary information

\begin{tabular}{|c|c|c|c|c|c|}
\hline Material & $\begin{array}{l}\text { Space } \\
\text { Group }\end{array}$ & $\begin{array}{l}\text { Structure } \\
\text { Type }\end{array}$ & $\begin{array}{l}\text { Structure } \\
\text { Reference }\end{array}$ & $\begin{array}{c}\text { LDA } \\
\text { gap [eV] }\end{array}$ & $\begin{array}{c}\text { Other } \\
\text { Reports }\end{array}$ \\
\hline $\mathrm{Ca}_{3} \mathrm{PbO}$ & P m -3 m & $\mathrm{CaTiO}_{3}$ & Widera \& Schaefer (1980) & 0.2 & $\begin{array}{l}\text { Examined further and verified by } \\
\text { Kariyado \& Ogata (2011) and } \\
\text { Sun, Chen, Yunoki, Li, \& Li (2010) }\end{array}$ \\
\hline $\mathrm{Sr}_{3} \mathrm{PbO}$ & $\mathrm{P} m-3 \mathrm{~m}$ & $\mathrm{CaTiO}_{3}$ & Widera \& Schaefer (1980) & 0.1 & $\begin{array}{l}\text { Examined further and verified by } \\
\text { Kariyado \& Ogata (2011) and } \\
\text { and Sun et al. (2010) }\end{array}$ \\
\hline $\mathrm{Ba}_{3} \mathrm{PbO}$ & $\mathrm{P} m-3 \mathrm{~m}$ & $\mathrm{CaTiO}_{3}$ & Widera \& Schaefer (1980) & 0.1 & $\begin{array}{c}\text { Examined further and verified by } \\
\text { Kariyado \& Ogata and (2011) } \\
\text { Sun et al. (2010) }\end{array}$ \\
\hline $\mathrm{Yb}_{3} \mathrm{PbO}$ & $\mathrm{P} \mathrm{m}-3 \mathrm{~m}$ & $\mathrm{CaTiO}_{3}$ & Velden \& Jansen (2004) & 0.2 & No further reports \\
\hline $\mathrm{Ca}_{3} \mathrm{SnO}$ & $\mathrm{P} m-3 \mathrm{~m}$ & $\mathrm{CaTiO}_{3}$ & Widera \& Schaefer (1980) & 0.2 & $\begin{array}{l}\text { Examined further and verified by } \\
\text { Kariyado \& Ogata (2011) and } \\
\text { Sun et al. (2010) }\end{array}$ \\
\hline $\mathrm{Sr}_{3} \mathrm{SnO}$ & $\mathrm{P}$ m $-3 \mathrm{~m}$ & $\mathrm{CaTiO}_{3}$ & Widera \& Schaefer (1980) & 0.1 & $\begin{array}{c}\text { Examined further and verified by } \\
\text { Kariyado \& Ogata (2011) and } \\
\text { Sun et al. (2010) }\end{array}$ \\
\hline $\mathrm{Yb}_{3} \mathrm{SnO}$ & $\mathrm{P} m-3 \mathrm{~m}$ & $\mathrm{CaTiO}_{3}$ & Velden \& Jansen (2004) & 0.1 & No further reports \\
\hline GdPtSb & $\mathrm{F}-43 \mathrm{~m}$ & AlLiSi & $\begin{array}{l}\text { de Vries, Thiel, \& Buschow } \\
\text { (1985) }\end{array}$ & 0.2 & No further reports \\
\hline $\mathrm{Bi}_{2} \mathrm{SeTe}_{2}$ & $\mathrm{R}-3 \mathrm{~m} \mathrm{H}$ & $\mathrm{Bi}_{2} \mathrm{Te}_{3}$ & Bland \& Basinski (1985) & 0.3 & Verified by Dai et al. (2012) \\
\hline $\mathrm{Bi}_{2} \mathrm{STe}_{2}$ & $\mathrm{R}-3 \mathrm{~m} \mathrm{H}$ & $\mathrm{Bi}_{2} \mathrm{Te}_{3}$ & Harker (1934) & 0.3 & No further reports \\
\hline $\mathrm{PbTl}_{4} \mathrm{Te}_{3}$ & I $4 / \mathrm{m} \mathrm{c} \mathrm{m}$ & $\mathrm{In}_{5} \mathrm{Bi}_{3}$ & $\begin{array}{l}\text { Bradtmoeller \& Boettcher } \\
\text { (1993) }\end{array}$ & 0.1 & Verified by Arpino (2014) \\
\hline $\mathrm{BiTl}_{9} \mathrm{Te}_{6}$ & I $4 / \mathrm{m} \mathrm{c} \mathrm{m}$ & $\mathrm{In}_{5} \mathrm{Bi}_{3}$ & Doert \& Boettcher (1994) & 0.1 & No further reports \\
\hline $\mathrm{BiTlTe}_{2}$ & $\mathrm{R}-3 \mathrm{~m} \mathrm{H}$ & $\mathrm{NaCrS}_{2}$ & Hockings \& White (1961) & $0.0 *$ & Verified by Chen et al. (2010) \\
\hline $\mathrm{SbTlTe}_{2}$ & $\mathrm{R}-3 \mathrm{~m} \mathrm{H}$ & $\mathrm{NaCrS}_{2}$ & Hockings \& White (1961) & 0.2 & No further reports \\
\hline $\mathrm{Bi}_{2} \mathrm{TeI}$ & $\mathrm{C} 12 / \mathrm{m} 1$ & $\mathrm{Bi}_{2} \mathrm{TeI}$ & Savilov et al. (2005) & 0.1 & No further reports \\
\hline $\mathrm{GeSb}_{4} \mathrm{Te}_{7}$ & $\mathrm{P}-3 \mathrm{~m} 1$ & $\mathrm{AgBiSe}_{2}$ & $\begin{array}{l}\text { Petrov, Imamov, \& Pinsker } \\
\text { (1968) }\end{array}$ & 0.2 & No further reports \\
\hline $\mathrm{HgKSb}$ & $\mathrm{P} 63 / \mathrm{m} \mathrm{m} \mathrm{c}$ & KZnAs & Vogel \& Schuster (1980) & 0.2 & No further reports \\
\hline
\end{tabular}

*The material has small hole pockets. 
For $\mathrm{Bi}_{2} \mathrm{SeTe}_{2}$, the electronic band gap closes without the spin-orbit term and the material appear to be a topological metal within the local density approximation (LDA). However, LDA is known to underestimate the electronic band gap and using higher order corrections such as GWA (Kotani \& Schilfgaarde, 2002) could very well open up a band gap, which can make it a topological insulator. We speculate that there are many not yet discovered topological metals, i.e. a bulk metal, but with topologically protected surface states. A data-mining search for these materials will be the subject for our next study.

$\mathrm{PbTl}_{4} \mathrm{Te}_{3}$ and $\mathrm{BiTl}_{9} \mathrm{Te}_{6}$ have a body-centered tetragonal Bravais lattice and belong to the $\mathrm{In}_{5} \mathrm{Bi}_{3}$ structure type family. $\mathrm{In}_{5} \mathrm{Bi}_{3}$ exhibit interesting electronic properties such as superconductivity. The two compounds both show the anti-crossing feature as spin-orbit is included and we note the curvature of these bands is smaller compared to, for example, the $\mathrm{Bi}_{2} \mathrm{Te}_{3}$ family. The indirect band gap is $0.1 \mathrm{eV}$, respectively.

$\mathrm{SbTlTe}_{2}$ and $\mathrm{BiTlTe}_{2}$ have the $\mathrm{NaCrS}_{2}$ structure type. The former material has an indirect band gap of $0.2 \mathrm{eV}$, while the latter has an electronic structure that is more complex. Using LDA methods, the material shows a small electron and hole pocket, but it could very well turn out to have a small band gap when investigated using higher order theory such as GWA.

The last three materials in Table 1 belong to different structure types and all have indirect band gaps, but with more exotic electronic band structures. $\mathrm{Bi}_{2} \mathrm{TeI}$ develop the anti-crossing feature at two high symmetry points $(\Gamma$ and $\mathrm{V})$ in the Brillouin zone. $\mathrm{GeSb}_{4} \mathrm{Te}_{7}$ and $\mathrm{HgKSb}$ show the band inversion at the A-point. The latter also displays a small hole pocket away from the A-point.

\section{Discussion}

There are two important takeaways from this study: 1) The determination of possible 3D topological insulators through a large scale analyses of DFT electronic structure calculations (discussed above). 2) The advancement of data-mining techniques for the use of determining exotic properties and pushing towards an effective predictive theory. This second point is particularly important given the major initiatives for predictive theory and materials design. Our goal to understand exotic phenomena is not limited to topological insulators and mainly uses them as an example of the power of data-mining application. This use of data-mining algorithms may be useful for identifying new thermoelectric, photovoltaic, or possibly superconducting materials. It is our goal to help broaden the search for new and exciting materials that exhibit exotic phenomena. However, there are main issues that challenge this process.

One issue is the lack of a large material properties databases that provide various electronic and structure properties for different materials. These databases are needed for large scale analyses of phenomena through parameter mapping. Recently, we have increased the aforementioned electronic structure database with the generation of a visual databases for material Fermi surfaces (Klintenberg, Haraldsen, \& Balatsky, 2013). The database is open to the public and can be utilized to look for many interesting phenomena (hot spots, electron and hole pockets, etc.), but having access to the data is not the only issue.

Another challenge is the need for a detailed understanding of the phenomena for effective parameter mapping. Parameter mapping is the algorithm that allows for a specific phenomena to be determined. For 3D topological insulators, it is the need for a distinct gap that would produce a band flip upon the presence of spin-orbit coupling. Therefore, to effectively investigate other material phenomena, one must understand that phenomena changes the structure, electronic bands, Fermi surface, or any other calculated property.

Our goal is to provide a general mechanism for determining the properties of complex materials using electronic structure. While in this work we only identify 17 candidates as possible topological insulators, many more are likely to have missed due to the narrow search parameters used in the calculations. A more extensive search over the same materials could lead to other topological insulators be realized. This method can also be adapted for a more in-depth and larger search. However, a larger search requires more computational power. Future research along this avenue can also look for phenomena that are not along symmetry directions.

Overall, there is a great need for predictive theory that can lead to understanding and designing of materials. In this work, we have used computing resources to data mine, search for, and identify potential new 3D topological insulators. In Table 1 , we present 17 materials that demonstrate anti-crossing features at the $\Gamma$-point $(\mathrm{V}$, A for the last three) when spin-orbit coupling is included. This feature is a standard band structure effect for topological insulators (Zhang et al., 2009b). As pointed out in reference (Lin et al., 2010), it is crucial to identify new groups of materials (electronic band structures) that are topologically non-trivial to maximize the likelihood for new physics 
as well as new devices (Fu \& Kane, 2009; Qi, Li, Zang, \& Zhang, 2009; Essin, Moore, \& Vanderbilt, 2009; Dzero, Sun, Galitski, \& Coleman, 2010; Linder, Tanaka, Yokoyama, Sudbo, \& Nagaosa, 2010; Ran, Yao, \& Vishwanath, 2010; Bonderson, Sarma, Freedman, \& Nayak, 2010). Since the first release of these results, a number of materials have been investigated further and some have been confirmed to be strong topological insulators. This establishes this data-mining method as a key component in the search for new and exotic materials, which may help reshape the materials science and initiatives like the materials gnome.

\section{Acknowledgments}

MK and AVB acknowledge support from the Swedish Research Council (VR), ERC-DM- 321031 and the Göran Gustafsson Stiftelse are acknowledged for financial support. JTH and AVB acknowledge support by BES at Los Alamos National Laboratory and Center for Integrated Nanotechnologies, an affirmative action equal opportunity employer, is operated by Los Alamos National Security, LLC, for the National Nuclear Security Administration of the U.S. Department of Energy under contract DE-AC52-06NA25396. AVB also acknowledges support from the Nordic Institute for Theoretical Physics (NORDITA).

M. K., J. T. H., and A. V. B. wrote the main manuscript text and J. T. H. prepared all figures. All authors reviewed the manuscript.

The authors declare no competing financial interests.

\section{References}

Arpino, K. E., Wallace, D. C., Nie, Y. F., Birol, T., King, P. D. C., Chatterjee, S., Uchida, M., .... McQueen, T. M. (2014). Evidence for Topologically Protected Surface States and a Superconducting Phase in $\left[\mathrm{Tl}_{4}\right]\left(\mathrm{Tl}_{1 ? x} \mathrm{Sn}_{x}\right) \mathrm{Te}_{3}$ Using Photoemission, Specific Heat, and Magnetization Measurements, and Density Functional Theory. Phys. Rev. Lett., 112, 017002 http://dx.doi.org/10.1103/PhysRevLett.112.017002

Benyelloul, K., \& Aourag, H. (2013). Bulk modulus prediction of austenitic stainless steel using a hybrid GAANN as a data mining tools. Comp. Mat. Sci., 77, 330. http://dx.doi.org/10.1016/j.commatsci.2013.04.058

Bland, J. A., \& Basinski, S. J. (1985). The crystal structure of $\mathrm{Bi}_{2} \mathrm{SeTe}_{2}$. Can. J. of Phys., 39, 1040-1043. http://dx.doi.org/10.1139/p61-113

Bonderson, P., Sarma, S. D., Freedman, M., \& Nayak, C. (2010). A blueprint for a topologically fault-tolerant quantum computer. ArXiv.1003.2856.

Bradtmoeller, S., \& Boettcher, P. (1993). Darstellung und Kristallstruktur von $\mathrm{SnTl}_{4} \mathrm{Te}_{3}$ und $\mathrm{PbTl}_{4} \mathrm{Te}_{3}$. Zeitschrift fuer Anorganische und Allgemeine Chemie., 619, 1155-1160. http://dx.doi.org/10.1002/zaac.19936190702

Buttiker, M. (2009). Edge-state physics without magnetic fields. Science, 325, 278. http://dx.doi.org/10.1126/science.1177157

Chen, Y. L., Analytis, J. G., Chu, J.-H., Liu, Z. K., Mo, S.-K., Qi, X. L., ... Shen Z.-X. (2009). Experimental realization of a three-dimensional topological insulator, BiTe. Science, $325,178$. http://dx.doi.org/10.1126/science.1173034

Chen, Y. L., Liu, Z. K., Analytis, J. G., Chu, J.-H., Zhang, H. J., Yan, B. H., ... Shen, Z.-X. (2010). Single Dirac Cone Topological Surface State and Unusual Thermoelectric Property of Compounds from a New Topological Insulator Family. Phys. Rev. Lett., 105, 266401. http://dx.doi.org/10.1103/PhysRevLett.105.266401

Collins, G. P. (2003). Computing with quantum knots. Sci. Am., 294, 57. http://dx.doi.org/10.1038/scientificamerican0406-56

Dai, X.-Q., Zhao, B., Zhao, J.-H., Li, Y.-H., Tang, Y.-N., \& Li N. (2012). Robust surface state of intrinsic topological insulator $\mathrm{Bi}_{2} \mathrm{Te}_{2} \mathrm{Se}$ thin films: A first-principles study. J. Phys.: Condens. Mat., 24, 035502. http://dx.doi.org/10.1088/0953-8984/24/3/035502

De Vries, J. W.,C., Thiel, R. C., \& Buschow, K. H. J. (1985). (155)Gd Moessbauer effect and Magnetic properties of some ternary gadolinium intermetallic compounds. J. Less-Common Met., 111, 313-320. http://dx.doi.org/10.1016/0022-5088(85)90203-6

Doert, T., \& Boettcher, P. (1994). Crystal structure of bismuth nonathallium hexatelluride, $\mathrm{BiTl}_{9} \mathrm{Te}_{6}$. Zeitschrift fuer Kristallographie, 209, 95-95. http://dx.doi.org/10.1524/zkri.1994.209.1.95

Dzero, M., Sun, K., Galitski, V., \& Coleman, P. (2010). Topological Kondo insulator. Phys. Rev. Lett., 104, 
106408. http://dx.doi.org/10.1103/PhysRevLett.104.106408

Essin, A., Moore, J. E., \& Vanderbilt, D. (2009). Magnetoelectric polarizability and axion electrodynamics in crystalline insulators. Phys. Rev. Lett., 102, 146805. http://dx.doi.org/10.1103/PhysRevLett.102.146805

Fisher, C. C., Tibbetts, K. J., Morgan, D., \& Ceder, G. (2006). Predicting crystal structure by merging data mining with quantum mechanics. Nature Materials, 5, 641. http://dx.doi.org/10.1038/nmat1691

Fu, L., \& Kane, C. L. (2009). Probing neutral Majorana fermion edge modes with charge transport. Phys. Rev. Lett., 102, 216403. http://dx.doi.org/10.1103/PhysRevLett.102.216403

Fu, L., Kane, C. L., \& Mele, E. J. (2007). Topological insulators in three dimensions. Phys. Rev. Lett., 98, 106803. http://dx.doi.org/10.1103/PhysRevLett.98.106803

Harker, D. (1934). The crystal structure of the mineral tetradymite, $\mathrm{Bi}_{2} \mathrm{Te}_{2} \mathrm{~S}$. Zeitschrift fuer Kristallographie, Kristallgeometrie, Kristallphysik, Kristallchemie, 89, 175-181. http://dx.doi.org/10.1524/zkri.1934.89.1.175

Hasan, M. Z., \& Kane, C. L. (2010). Topological insulators. Rev. Mod. Phys., 82, 3045. http://dx.doi.org/10.1103/RevModPhys.82.3045

Hazen, R. M. (1988). Perovskites. Sci. Am., 258, 74. http://dx.doi.org/10.1038/scientificamerican0688-74

Hockings, E. F., \& White, J. G. (1961). The crystal structures of $\mathrm{TlSbTe}_{2}$ and $\mathrm{TlBiTe}_{2}$. Acta Crystallographica, 14, 328-328. http://dx.doi.org/10.1107/S0365110X61001108

Hsieh, D., Qian, D., Wray, L., Xia, Y., Hor, Y. S., Cava, R. J., \& Hasan, M. Z. (2008). A topological Dirac insulator in a quantum spin Hall phase. Nature, 452, 970. http://dx.doi.org/10.1038/nature06843

Hsieh, D., Xia, Y., Wray, L., Qian, D., Pal, A., Dil, J. H., ... Hasan, M. Z. (2009). Observation of unconventional quantum spin textures in topological insulators. Science, 323, 919. http://dx.doi.org/10.1126/science.1167733

Kane, C. L. (2008). An insulator with a twist. Nature Phys., 4, 348. http://dx.doi.org/10.1038/nphys955

Kane, C. L., \& Mele, E. J. (2005a). Quantum spin Hall effect in graphene. Phys. Rev. Lett., 95, 226801. http://dx.doi.org/10.1103/PhysRevLett.95.226801

Kane, C. L., \& Mele, E. J. (2005b). $Z_{2}$ topological order and the quantum spin Hall effect. Phys. Rev. Lett., 95, 146802. http://dx.doi.org/10.1103/PhysRevLett.95.146802

Kariyado, T. \& Ogata, M. (2011). Three-Dimensional Dirac Electrons at the Fermi Energy in Cubic Inverse Perovskites: $\mathrm{Ca}_{3} \mathrm{PbO}$ and Its Family. J. Phys. Soc. Japan, 80, 083704. http://dx.doi.org/10.1143/JPSJ.80.083704

Klintenberg, M. (2010). The search for strong topological insulators. ArXiv:1007.4838.

Klintenberg, M. (2014). Electronic Structure Database. Retrieved on January 6th, 2014 from www.materialsgenome.se

Klintenberg, M., \& Eriksson, O. (2013). Possible high-temperature superconductors predicted from electronic structure and data-filtering algorithms. Comp. 282. http://dx.doi.org/10.1016/j.commatsci.2012.08.038

Klintenberg, M., Derenso, S. E., \& Weber, M. J. (2002a). Potential new scintillators identified by electronic structure calculations. Nucl. Inst. Meth., A486, 298-302. http://dx.doi.org/10.1016/S0168-9002(02)00723-4

Klintenberg, M., Derenso, S. E., \& Weber, M. J. (2002b). A systematic search for new scintillators using electronic structure calculations, Nanotech: Technical Proceedings of the 2002 International Conference on Computational Nanoscience and Nanotechnology, 2.

Klintenberg, M., Haraldsen, J. T., \& Balatsky, A. V. (2013). Unpublished Data; 3D Fermi Surface Database, Retrieved from http://cint.lanl.gov/viz2.shtml or http://gurka.fysik.uu.se/esp-fs/; A joint project between Uppsula University and the Center for Integrated Nanotechnologies at Los Alamos National Laboratory. July 28th, 2013.

Kotani, T., \& Schilfgaarde, M. (2002). All-electron GW approximation with the mixed basis expansion based on the full-potential LMTO method. Solid State Commun., 121, 461. http://dx.doi.org/10.1016/S00381098(02)00028-5

Lebegue, S., Bj orkman, T., Klintenberg, M., Nieminen, R. M., \& Eriksson, O. (2013). Two-dimensional materials 
from data mining and ab-initio calculations. Phys. Rev. $\quad$ X, 3, 031002 . http://dx.doi.org/10.1103/PhysRevX.3.031002

Lin, H., Wray, L. A., Xia, Y., Xu, S., Jia, S., Cava, J. R., ... Hasan, M. Z. (2010). Half-Heusler ternary compounds as new multifunctional experimental platforms for topological quantum phenomena. Nature Matr., 9, 546549. http://dx.doi.org/10.1038/nmat2771

Linder, J., Tanaka, Y., Yokoyama, T., Sudbo, A., \& Nagaosa, N. (2010). Unconventional superconductivity on a topological insulator. Phys. Rev. Lett., 104, 067001. http://dx.doi.org/10.1103/PhysRevLett.104.067001

Materials Genome Initiative for Global Competitiveness. (2014). Materials Gnome Initiative. Retrieved on January 6th, 2014 from http://www.whitehouse.gov/mgi

Moore, J. E. (2009a). An insulator metallic side. Nature, 460, 1090. http://dx.doi.org/10.1038/4601090b

Moore, J. E. (2009b). Topological insulators: The next generation. Nature Phys., 5, 378. http://dx.doi.org/10.1038/nphys1294

Moore, J. E. (2010). The birth of topological insulators. Nature, 464, 194. http://dx.doi.org/10.1038/nature08916

Moore, J. E., \& Bals, L. (2007). Topological invariants of time-reversal-invariant band structures. Phys. Rev. B, 75, 121306(R). http://dx.doi.org/10.1103/PhysRevB.75.121306

Morales, M. A., McMahon, J. M., Pierleoni, C., \& Ceperley, D. M. (2013). Towards a predictive first-principles description of solid molecular hydrogen with density functional theory. Phys. Rev. B, 87, 184107. http://dx.doi.org/10.1103/PhysRevB.87.184107

Murakami, S., Nagaosa, N., \& Zhang, S. C. (2004). Spin-Hall insulator. Phys. Rev. Lett., 93, 156804. http://dx.doi.org/10.1103/PhysRevLett.93.156804

Olson, G. B. (2013). Genomic materials design: The ferrous frontier. Acta Materialia, 61, 771. http://dx.doi.org/10.1016/j.actamat.2012.10.045

Ortiz, C., Eriksson, O., \& Klintenberg, M. (2009). Data mining and accelerated electronic structure theory as a tool in the search for new functional materials. Comput. Matr. Sci., 44, 1042. http://dx.doi.org/10.1016/j.commatsci.2008.07.016

Petrov, I. I., Imamov, R. M., \& Pinsker, Z. G. (1968). Electron-diffraction determination of the structures of $\mathrm{Ge}_{2} \mathrm{Sb}_{2} \mathrm{Te}_{5}$ and $\mathrm{GeSb}_{4} \mathrm{Te}_{7}$. Kristallografiya, 13, 417-421.

Qi, X.-L., Li R., Zang J., Zhang S.-L. (2009). Inducing a magnetic monopole with topological surface states. Science, 323, 1184-1187. http://dx.doi.org/10.1126/science.1167747

Ran, Y., Yao, H., \& Vishwanath, A. (2010) Composite fermions and quantum Hall stripes on the topological insulator surface. ArXiv.1003.0901.

Roushan, P., Seo, J., Parker, C. V., Hor, Y. S., Hsieh, D., Qian, D., ... Yazdani, A. (2009). Topological surface states protected from backscattering by chiral spin texture. Nature, 460, 1106-1109. http://dx.doi.org/10.1038/nature08308

Roy, R. (2009). Topological phases and the quantum spin Hall effect in three dimensions. Phys. Rev. B, 79, 195322. http://dx.doi.org/10.1103/PhysRevB.79.195322

Savilov, S. V., Khrustalev, V. N., Kuznetsov, A. N., Popovkin, B. A., \& Antipin, M. (2005). New subvalent bismuth telluroiodides incorporating $\mathrm{Bi}_{2}$ layers: The crystal and electronic structure of $\mathrm{Bi}_{2}$ TeI. Izvestiya Akad. Nauk, Seriya Khimicheskaya, 54, 86-91. http://dx.doi.org/10.1007/s11172-005-0221-8

Seshadri, R., Brock, S. L., Ramirez, A., Subramanian, M. A., \& Thompson, M. E. (2012). Advances in the development and growth of functional materials: Toward the paradigm of materials by design. Mat. Res. Bull., 37, 682. http://dx.doi.org/10.1557/mrs.2012.147

Skriver, H. L. (1984). The LMTO Method: Muffin-Tin Orbitals and Electronic Structure. Berlin: Springer.

Sun, S., Chen, X.-Q., Yunoki, S., Li, D., \& Li, Y. (2010). New Family of Three-Dimensional Topological Insulators with Antiperovskite Structure. Phys. Rev. http://dx.doi.org/10.1103/PhysRevLett.105.216406 
Velden, A., \& Jansen, M. (2004). Zur Kenntnis der inversen Perovskite M3 T O (M = Ca, Sr, Yb; T = Si, Ge, Sn, $\mathrm{Pb}$ ). Zeitschrift fuer Anorganische und Allgemeine Chemie., 630, 234-238. http://dx.doi.org/10.1002/zaac.200300313

Vogel, P., \& Schuster, H. U. (1980). Ternaere Verbindungen mit modifizierter $\mathrm{Ni}_{2}$ In Struktur. Zeitschrift fuer Naturforschung, Teil B. Anorganische Chemie, Organische Chemie, 35, 114-116.

Widera, A., \& Schaefer, H. (1980). Uebergangsformen zwischen den Zintlphasen und echten Salzen: die Verbindungen $\mathrm{A} 3 \mathrm{~B} \mathrm{O}$ mit $\mathrm{A}=\mathrm{Ca}, \mathrm{Sr}, \mathrm{Ba}$ und $\mathrm{B}=\mathrm{Sn}, \mathrm{Pb}$. Mat. Res. Bull., 15, 1805-1809. http://dx.doi.org/10.1016/0025-5408(80)90200-7

Xia, Y., Qian, D., Hsieh, D., Wray, L., Pal, A., Lin, H., ... Hasan, M. Z. (2009). Observation of a large-gap topological-insulator class with a single Dirac cone on the surface. Nature Phys., 5, 398. http://dx.doi.org/10.1038/nphys1274

Yang, K., Setyawan, W., Wang, S., Buongiorno Nardelli, M., \& Curtarolo, S. (2012). A search model for topological insulators with high-throughput robustness descriptors, Nature Materials, 11, 614-619. http://dx.doi.org/10.1038/nmat3332

Zhang, H., Liu, C.-X, Qi, X.-L., Dai, X., Fang, Z., \& Zhang, S.-C. (2009). Topological insulators in Bi2Se3, Bi2Te3 and Sb2Te3 with a single Dirac cone on the surface. Nature Phys., 5, 438. http://dx.doi.org/10.1038/nphys 1270

\section{Copyrights}

This material is declared a work of the U. S. Government and is not subject to copyright protection in the United States, with first publication rights granted to the journal.

This is an open-access article distributed under the terms and conditions of the Creative Commons Attribution license (http://creativecommons.org/licenses/by/3.0/). 